\title{
A System of Random Nonlinear Variational Inclusions Involving Random Fuzzy Mappings and $H(\cdot, \cdot)$-Monotone Set-Valued Mappings
}

\author{
Xin-kun Wu and Yun-zhi Zou \\ College of Mathematics, Sichuan University, Chengdu, Sichuan 610064, China \\ Correspondence should be addressed to Yun-zhi Zou, zouyz@scu.edu.cn \\ Received 8 June 2010; Accepted 24 July 2010 \\ Academic Editor: Qamrul Hasan Ansari
}

Copyright (C) 2010 X.-k. Wu and Y.-z. Zou. This is an open access article distributed under the Creative Commons Attribution License, which permits unrestricted use, distribution, and reproduction in any medium, provided the original work is properly cited.

We introduce and study a new system of random nonlinear generalized variational inclusions involving random fuzzy mappings and set-valued mappings with $H(\cdot, \cdot)$-monotonicity in two Hilbert spaces and develop a new algorithm which produces four random iterative sequences. We also discuss the existence of the random solutions to this new kind of system of variational inclusions and the convergence of the random iterative sequences generated by the algorithm.

\section{Introduction}

The classic variational inequality problem $\operatorname{VI}(F, K)$ is to determine a vector $x^{*} \in K \subset R^{n}$, such that

$$
\left\langle F\left(x^{*}\right)^{T}, x-x^{*}\right\rangle \geq 0, \quad \forall x \in K
$$

where $F$ is a given continuous function from $K$ to $R^{n}$ and $K$ is a given closed convex subset of the $n$-dimensional Euclidean space $R^{n}$. This is equivalent to find an $x^{*} \in K$, such that

$$
0 \in F\left(x^{*}\right)+N_{\perp}\left(x^{*}\right)
$$

where $N_{\perp}$ is normal cone operator.

Due to its enormous applications in solving problems arising from the fields of economics, mechanics, physical equilibrium analysis, optimization and control, transportation 
equilibrium, and linear or nonlinear programming etcetera, variational inequality and its generalizations have been extensively studied during the past 40 years. For details, we refer readers to [1-7] and the references therein.

It is not a surprise that many practical situations occur by chance and so variational inequalities with random variables/mappings have also been widely studied in the past decade. For instance, some random variational inequalities and random quasivariational inequalities problems have been introduced and studied by Chang [8], Chang and Huang $[9,10]$, Chang and Zhu [11], Huang [12, 13], Husain et al. [14], Tan et al. [15], Tan [16], and Yuan [7].

It is well known that one of the most important and interesting problems in the theory of variational inequalities is to develop efficient and implementable algorithms for solving variational inequalities and its generalizations. The monotonic properties of associated operators play essential roles in proving the existence of solutions and the convergence of sequences generated by iterative algorithms. In 2001, Huang and Fang [17] were the first to introduce the generalized $m$-accretive mapping and give the definition of the resolvent operator for generalized $m$-accretive mappings in Banach spaces. They also showed some properties of the resolvent operator for generalized $m$-accretive mappings. Recently, Fang and Huang, Verma, and Cho and Lan investigated many generalized operators such as $\mathrm{H}$ monotone, $H$-accretive, $(H, \eta)$-monotone, $(H, \eta)$-accretive, and $(A, \eta)$-accretive mappings. For details, we refer to [6, 17-22] and the references therein. In 2008, Zou and Huang [23] introduced the $H(\cdot, \cdot)$-accretive operator in Banach spaces which provides a unified framework for the existing $H$-monotone, $(H, \eta)$-monotone, and $(A, \eta)$-monotone operators in Hilbert spaces and $H$-accretive, $(H, \eta)$-accretive, and $(A, \eta)$-accretive operators in Banach spaces.

In 1965, Zadeh [24] introduced the concept of fuzzy sets, which became a cornerstone of modern fuzzy mathematics. To explore connections among VIs, fuzzy mapping and random mappings, in 1997, Huang [25] introduced the concept of random fuzzy mappings and studied the random nonlinear quasicomplementarity problem for random fuzzy mappings. Later, Huang [26] studied the random generalized nonlinear variational inclusions for random fuzzy mappings. In 2005, Ahmad and Bazán [27] studied a class of random generalized nonlinear mixed variational inclusions for random fuzzy mappings and constructed an iterative algorithm for solving such random problems. For related work in this hot area, we refer to Ahmad and Farajzadeh [28], Ansari and Yao [29], Chang and Huang [9, 10], Cho and Huang [30], Cho and Lan [31], Huang [25, 26, 32], Huang et al. [33], and the references therein.

Motivated and inspired by recent research work mentioned above in this field, in this paper, we try to inject some new energy into this interesting field by studying on a new kind of random nonlinear variational inclusions in two Hilbert spaces. We will prove the existence of random solutions to the system of inclusions and propose an algorithm which produces a convergent iterative sequence. For a suitable choice of some mappings, we can obtain several known results $[10,11,21,23,31,34]$ as special cases of the main results of this paper.

\section{Preliminaries}

Throughout this paper, let $(\Omega, \mathcal{A})$ be a measurable space, where $\Omega$ is a set and $\mathcal{A}$ is a $\sigma$-algebra over $\Omega$. Let $X_{1}$ be a separable real Hilbert space endowed with a norm $\|\cdot\|_{X_{1}}$ and an inner product $\langle\cdot, \cdot\rangle_{X_{1}}$. Let $X_{2}$ be a separable real Hilbert space endowed with a norm $\|\cdot\|_{X_{2}}$ and an inner product $\langle\cdot, \cdot\rangle_{X_{2}}$. 
We denote by $D(\cdot, \cdot)$ the Hausdorff metric between two nonempty closed bounded subsets, where the Hausdorff metric between $A$ and $B$ is defined by

$$
D(A, B)=\max \left\{\sup _{a \in A} \inf _{b \in B} d(a, b), \sup _{b \in B} \inf _{a \in A} d(a, b)\right\} .
$$

We denote by $B\left(X_{1}\right), 2^{X_{1}}$, and $C B\left(X_{1}\right)$ the class of Borel $\sigma$-fields in $X_{1}$, and the family of all nonempty subsets of $X_{1}$, the family of all nonempty closed bounded subsets of $X_{1}$.

In this paper, to make it self-contained, we start with the following basic definitions and similar definitions can also be found in $[26,32,34]$.

Definition 2.1. A mapping $x_{1}: \Omega \rightarrow X_{1}$ is said to be measurable if for any $B \in B\left(X_{1}\right)$,

$$
\{t \in \Omega: x(t) \in B\} \in \mathcal{A} .
$$

Definition 2.2. A mapping $T_{1}: \Omega \times X_{1} \rightarrow X_{1}$ is called a random mapping if for any $x \in X_{1}$, $z_{1}(t)=T_{1}(t, x)$ is measurable.

Definition 2.3. A random mapping $T_{1}: \Omega \times X_{1} \rightarrow X_{1}$ is said to be continuous if for any $t \in \Omega$, $T_{1}(t, \cdot): X_{1} \rightarrow X_{1}$ is continuous.

Definition 2.4. A set-valued mapping $V_{1}: \Omega \rightarrow 2^{X_{1}}$ is said to be measurable if for any $B \in$ $B\left(X_{1}\right)$,

$$
V_{1}^{-1}(B)=\left\{v \in \Omega: V_{1}(v) \cap B \neq \emptyset\right\} \in \mathcal{A} .
$$

Definition 2.5. A mapping $u: \Omega \rightarrow X_{1}$ is called a measurable selection of a set-valued measurable mapping $U: \Omega \rightarrow 2^{X_{1}}$ if $u$ is measurable and for any $t \in \Omega, u(t) \in U(t)$.

Definition 2.6. A set-valued mapping $W_{1}: \Omega \times X_{1} \rightarrow 2^{X_{1}}$ is called random set-valued if for any $x_{1} \in X_{1}, W_{1}\left(\cdot, x_{1}\right): \Omega \rightarrow 2^{X_{1}}$ is a measurable set valued mapping.

Definition 2.7. A random set-valued mapping $W_{1}: \Omega \times X_{1} \rightarrow C B\left(X_{1}\right)$ is said to be $\xi_{E}(t)-D$ continuous if there exists a measurable function $\xi_{E}: \Omega \rightarrow(0,+\infty)$, such that

$$
D\left(W_{1}\left(t, x_{1}(t)\right), W_{1}\left(t, x_{2}(t)\right)\right) \leq \xi_{E}(t)\left\|x_{1}(t)-x_{2}(t)\right\|_{X_{1}},
$$

for all $t \in \Omega$ and $x_{1}(t), x_{2}(t) \in X_{1}$.

Definition 2.8. A set-valued mapping $A: X_{1} \rightarrow 2^{X_{1}}$ is said to be monotone if for all $x_{1}, y_{1} \in X_{1}$ and $u_{1} \in A\left(x_{1}\right), v_{1} \in A\left(y_{1}\right)$,

$$
\left\langle u_{1}-v_{1}, x_{1}-y_{1}\right\rangle_{X_{1}} \geq 0
$$

Definition 2.9. Let $f_{1}, g_{1}: X_{1} \rightarrow X_{1}$ and $H_{1}: X_{1} \times X_{1} \rightarrow X_{1}$ be three single-valued mappings and $A: X_{1} \rightarrow 2^{X_{1}}$ be a set-valued mapping. $A$ is said to be $H_{1}(\cdot, \cdot)$-monotone with respect to operators $f_{1}$ and $g_{1}$ if $\mathrm{A}$ is monotone and $\left(H_{1}\left(f_{1}, g_{1}\right)+\lambda A\right)\left(X_{1}\right)=X_{1}$, for every $\lambda>0$. 
Definition 2.10. The inverses of $A: X_{1} \rightarrow 2^{X_{1}}$ and $B: X_{2} \rightarrow 2^{X_{2}}$ are defined as follows, respectively,

$$
\begin{array}{ll}
A^{-1}(y)=\left\{x \in X_{1}: y \in A(x)\right\}, & \forall y \in X_{1}, \\
B^{-1}(y)=\left\{x \in X_{2}: y \in B(x)\right\}, & \forall y \in X_{2} .
\end{array}
$$

Definition 2.11. $p: \Omega \times X_{1} \rightarrow X_{1}$ is said to be

(1) monotone if

$$
\left\langle p\left(t, x_{1}(t)\right)-p\left(t, x_{2}(t)\right), x_{1}(t)-x_{2}(t)\right\rangle_{X_{1}} \geq 0, \quad \forall t \in \Omega, \forall x_{1}(t), \quad x_{2}(t) \in X_{1}
$$

(2) strictly monotone if $p$ is monotone and

$$
\left\langle p\left(t, x_{1}(t)\right)-p\left(t, x_{2}(t)\right), x_{1}(t)-x_{2}(t)\right\rangle_{X_{1}}=0 \Longleftrightarrow x_{1}(t)=x_{2}(t), \quad \forall t \in \Omega, \forall x_{1}(t), x_{2}(t) \in X_{1},
$$

(3) $\delta_{p}(t)$-strongly monotone if there exists some measurable function $\delta_{p}: \Omega \rightarrow$ $(0,+\infty)$, such that

$$
\left\langle p\left(t, x_{1}(t)\right)-p\left(t, x_{2}(t)\right), x_{1}(t)-x_{2}(t)\right\rangle_{X_{1}} \geq \delta_{p}(t)\left\|x_{1}(t)-x_{2}(t)\right\|_{x_{1}}^{2}, \quad \forall t \in \Omega, \forall x_{1}(t), x_{2}(t) \in X_{1},
$$

(4) $\sigma_{p}(t)$-Lipschitz continuous if there exists some measurable function $\sigma_{p}: \Omega \rightarrow$ $(0,+\infty)$, such that

$$
\left\|p\left(t, x_{1}(t)\right)-p\left(t, x_{2}(t)\right)\right\|_{X_{1}} \leq \sigma_{p}(t)\left\|x_{1}(t)-x_{2}(t)\right\|_{X_{1}}, \quad \forall t \in \Omega, \quad \forall x_{1}(t), x_{2}(t) \in X_{1} .
$$

Definition 2.12. A single-valued mapping $M: X_{1} \times X_{1} \times X_{2} \rightarrow X_{1}$ is said to be

(1) $\zeta_{A}(t)$-strongly monotone with respect to the random single-valued mapping $s_{M}$ : $\Omega \times X_{1} \rightarrow X_{1}$ in the first argument if there exists some measurable function $\zeta_{A}$ : $\Omega \rightarrow(0,+\infty)$, such that

$$
\left\langle M\left(s_{M}\left(t, u_{1}(t)\right), \cdot, \cdot\right)-M\left(s_{M}\left(t, u_{2}(t)\right), \cdot, \cdot\right), u_{1}(t)-u_{2}(t)\right\rangle_{X_{1}} \geq \zeta_{A}(t)\left\|u_{1}(t)-u_{2}(t)\right\|_{X_{1}}^{2},
$$

for all $t \in \Omega$ and $u_{1}(t), u_{2}(t) \in X_{1}$,

(2) $\xi_{M}(t)$-Lipschitz continuous with respect to the random single-valued mapping $s_{M}$ : $\Omega \times X_{1} \rightarrow X_{1}$ in its first argument if there exists some measurable function $\xi_{M}$ : $\Omega \rightarrow(0,+\infty)$, such that

$$
\left\|M\left(s_{M}\left(t, u_{1}(t)\right), \cdot, \cdot\right)-M\left(s_{M}\left(t, u_{2}(t)\right), \cdot, \cdot\right)\right\|_{X_{1}} \leq \xi_{M}(t)\left\|u_{1}(t)-u_{2}(t)\right\|_{X_{1}},
$$

for all $t \in \Omega$ and $u_{1}(t), u_{2}(t) \in X_{1}$, 
(3) $\beta_{M}(t)$-Lipschitz continuous with respect to its second argument if there exists some measurable function $\beta_{M}: \Omega \rightarrow(0,+\infty)$, such that

$$
\left\|M\left(\cdot, x_{1}(t), \cdot\right)-M\left(\cdot, x_{2}(t), \cdot\right)\right\|_{X_{1}} \leq \beta_{M}(t)\left\|x_{1}(t)-x_{2}(t)\right\|_{X_{1}},
$$

for all $t \in \Omega$ and $x_{1}(t), x_{2}(t) \in X_{1}$,

(4) $\eta_{M}(t)$-Lipschitz continuous with respect to its third argument if there exists some measurable function $\eta_{M}: \Omega \rightarrow(0,+\infty)$ such that

$$
\left\|M\left(\cdot, \cdot, y_{1}(t)\right)-M\left(\cdot, \cdot, y_{2}(t)\right)\right\|_{X_{1}} \leq \eta_{M}(t)\left\|y_{1}(t)-y_{2}(t)\right\|_{X_{2}}
$$

for all $t \in \Omega$ and $y_{1}(t), y_{2}(t) \in X_{2}$;

Definition 2.13. Assume that $p: \Omega \times X_{1} \rightarrow X_{1}$ is a random single-valued mapping, $f_{1}: X_{1} \rightarrow$ $X_{1}, g_{1}: X_{1} \rightarrow X_{1}$, and $H_{1}\left(f_{1}, g_{1}\right): X_{1} \rightarrow X_{1}$ are three single-valued mappings, $H_{1}\left(f_{1}, g_{1}\right)$ is said to be

(1) $\mu_{A}(t)$-strongly monotone with respect to the mapping $p$ if there exists some measurable function $\mu_{A}: \Omega \rightarrow(0,+\infty)$ such that

$$
\begin{aligned}
& \left\langle H_{1}\left(f_{1}\left(p\left(t, x_{1}(t)\right)\right), g_{1}\left(p\left(t, x_{1}(t)\right)\right)\right)-H_{1}\left(f_{1}\left(p\left(t, y_{1}(t)\right)\right), g_{1}\left(p\left(t, y_{1}(t)\right)\right)\right), x_{1}(t)-y_{1}(t)\right\rangle_{X_{1}} \\
& \geq \mu_{A}(t)\left\|x_{1}(t)-y_{1}(t)\right\|_{X_{1}{ }^{\prime}}^{2}
\end{aligned}
$$

for all $t \in \Omega$ and $x_{1}(t), y_{1}(t) \in X_{1}$,

(2) $a_{A}(t)$-Lipschitz continuous with respect to the mapping $p$ if there exists some measurable function $a_{A}: \Omega \rightarrow(0,+\infty)$ such that

$$
\begin{aligned}
& \left\|H_{1}\left(f_{1}\left(p\left(t, x_{1}(t)\right)\right), g_{1}\left(p\left(t, x_{1}(t)\right)\right)\right)-H_{1}\left(f_{1}\left(p\left(t, y_{1}(t)\right)\right), g_{1}\left(p\left(t, y_{1}(t)\right)\right)\right)\right\|_{X_{1}} \\
& \leq a_{A}(t)\left\|x_{1}(t)-y_{1}(t)\right\|_{X_{1}{ }^{\prime}}
\end{aligned}
$$

for all $t \in \Omega$ and $x_{1}(t), y_{1}(t) \in X_{1}$.

(3) $\alpha_{A}$-strongly monotone with respect to $f_{1}$ in the first argument if there exists a positive constant $\alpha_{A}$, such that

$$
\left\langle H_{1}\left(f_{1}\left(x_{1}\right), u_{1}\right)-H_{1}\left(f_{1}\left(y_{1}\right), u_{1}\right), x_{1}-y_{1}\right\rangle_{X_{1}} \geq \alpha_{A}\left\|x_{1}-y_{1}\right\|_{X_{1}}^{2}
$$

for all $x_{1}, y_{1}, u_{1} \in X_{1}$, 
(4) $\beta_{A}$-relaxed monotone with respect to $g_{1}$ in the second argument if there exists a positive constant $\beta_{A}$, such that

$$
\left\langle H_{1}\left(u_{1}, g_{1}\left(x_{1}\right)\right)-H_{1}\left(u_{1}, g_{1}\left(y_{1}\right)\right), x_{1}-y_{1}\right\rangle_{X_{1}} \geq-\beta_{A}\left\|x_{1}-y_{1}\right\|_{X_{1}}^{2}
$$

for all $x_{1}, y_{1}, u_{1} \in X_{1}$.

Let $\mathcal{F}\left(X_{1}\right)$ be a collection of all fuzzy sets over $X_{1}$. A mapping $F$ from $\Omega$ into $\mathcal{F}\left(X_{1}\right)$ is called a fuzzy mapping. If $F$ is a fuzzy mapping on $X_{1}$, then for any given $t \in \Omega, F(t)$ (denote it by $F_{t}$ in the sequel) is a fuzzy set on $X_{1}$ and $F_{t}(y)$ is the membership function of $y$ in $F_{t}$.

Let $A \in \mathcal{F}\left(X_{1}\right), \alpha \in[0,1]$, then the set

$$
(A)_{\alpha}=\left\{x \in X_{1}: A(x) \geq \alpha\right\}
$$

is called an $\alpha$-cut set of fuzzy set $A$.

Definition 2.14. A random fuzzy mapping $F: \Omega \rightarrow \mathcal{F}\left(X_{1}\right)$ is said to be measurable if for any given $\alpha \in(0,1],(F(\cdot))_{\alpha}: \Omega \rightarrow 2^{X_{1}}$ is a measurable set-valued mapping.

Definition 2.15. A fuzzy mapping $E: \Omega \times X_{1} \rightarrow \mathcal{F}\left(X_{1}\right)$ is called a random fuzzy mapping if for any given $x_{1} \in X_{1}, E\left(\cdot, x_{1}\right): \Omega \rightarrow \mathcal{F}\left(X_{1}\right)$ is a measurable fuzzy mapping.

Remark 2.16. The above is mainly about some definitions in $X_{1}$. There are similar definitions and notations for operators in $X_{2}$.

Let $E: \Omega \times X_{1} \rightarrow \mathcal{F}\left(X_{1}\right)$ and $F: \Omega \times X_{2} \rightarrow \mathcal{F}\left(X_{2}\right)$ be two random fuzzy mappings satisfying the following condition $(* *)$ :

$(* *)$ there exist two mappings $\alpha: X_{1} \rightarrow(0,1]$ and $\beta: X_{2} \rightarrow(0,1]$, such that

$$
\begin{aligned}
& \left(E_{t, x_{1}}\right)_{\alpha\left(x_{1}\right)} \in C B\left(X_{1}\right), \quad \forall\left(t, x_{1}\right) \in \Omega \times X_{1}, \\
& \left(F_{t, x_{2}}\right)_{\beta\left(x_{2}\right)} \in C B\left(X_{2}\right), \quad \forall\left(t, x_{2}\right) \in \Omega \times X_{2} .
\end{aligned}
$$

By using the random fuzzy mappings $E$ and $F$, we can define the two set-valued mappings $E^{*}$ and $F^{*}$ as follows, respectively,

$$
\begin{array}{ll}
E^{*}: \Omega \times X_{1} \longrightarrow C B\left(X_{1}\right), \quad\left(t, x_{1}\right) \longrightarrow\left(E_{t, x_{1}}\right)_{\alpha\left(x_{1}\right)}, \quad \forall\left(t, x_{1}\right) \in \Omega \times X_{1}, \\
F^{*}: \Omega \times X_{2} \longrightarrow C B\left(X_{2}\right), \quad\left(t, x_{2}\right) \longrightarrow\left(E_{t, x_{2}}\right)_{\alpha\left(x_{2}\right)}, \quad \forall\left(t, x_{2}\right) \in \Omega \times X_{2} .
\end{array}
$$

It follows that

$$
\begin{aligned}
& E^{*}\left(t, x_{1}\right)=\left(E_{t, x_{1}}\right)_{\alpha\left(x_{1}\right)}=\left\{z_{1} \in X_{1}:\left(E_{t, x_{1}}\right)\left(z_{1}\right) \geq \alpha\left(x_{1}\right)\right\} \\
& F^{*}\left(t, x_{2}\right)=\left(F_{t, x_{2}}\right)_{\beta\left(x_{2}\right)}=\left\{z_{2} \in X_{2}:\left(F_{t, x_{2}}\right)\left(z_{2}\right) \geq \beta\left(x_{2}\right)\right\} .
\end{aligned}
$$

It is easy to see that $E^{*}$ and $F^{*}$ are two random set-valued mappings. We call $E^{*}$ and $F^{*}$ the random set-valued mappings induced by the fuzzy mappings $E$ and $F$, respectively. 
Problem 1. Let $f_{1}, g_{1}: X_{1} \rightarrow X_{1}$ be two single-valued mappings and $s_{M}, p: \Omega \times X_{1} \rightarrow X_{1}$ be two random single-valued mappings. Let $f_{2}, g_{2}: X_{2} \rightarrow X_{2}$ be two single-valued mappings and $s_{N}, q: \Omega \times X_{2} \rightarrow X_{2}$ be two random single-valued mappings. Let $H_{1}: X_{1} \times X_{1} \rightarrow X_{1}$, $H_{2}: X_{2} \times X_{2} \rightarrow X_{2}, M: X_{1} \times X_{1} \times X_{2} \rightarrow X_{1}$ and $N: X_{2} \times X_{1} \times X_{2} \rightarrow X_{2}$ be four singlevalued mappings. Suppose that $A: X_{1} \rightarrow 2^{X_{1}}$ is an $H_{1}(\cdot, \cdot)$-monotone mapping with respect to $f_{1}$ and $g_{1}$ and $B: X_{2} \rightarrow 2^{X_{2}}$ is an $H_{2}(\cdot, \cdot)$-monotone mapping with respect to $f_{2}$ and $g_{2}$. $E: \Omega \times X_{1} \rightarrow \mathcal{F}\left(X_{1}\right)$ and $F: \Omega \times X_{2} \rightarrow \mathcal{F}\left(X_{2}\right)$ are two random fuzzy mappings, $\alpha, \beta, E^{*}$, and $F^{*}$ are the same as the above. Assume that $p(t, u(t)) \cap \operatorname{dom}(A) \neq \emptyset$ and $q(t, v(t)) \cap \operatorname{dom}(B) \neq \emptyset$ for all $t \in \Omega$. We consider the following problem.

Find four measurable mappings $u, x: \Omega \rightarrow X_{1}$ and $v, y: \Omega \rightarrow X_{2}$, such that

$$
\begin{gathered}
E_{t, u(t)}(x(t)) \geq \alpha(u(t)), \\
F_{t, v(t)}(y(t)) \geq \beta(v(t)), \\
0 \in M\left(s_{M}(t, u(t)), x(t), y(t)\right)+A(p(t, u(t))), \\
0 \in N\left(s_{N}(t, v(t)), x(t), y(t)\right)+B(q(t, v(t))),
\end{gathered}
$$

for all $t \in \Omega$.

Problem 1 is called a system of generalized random nonlinear variational inclusions involving random fuzzy mappings and set-valued mappings with $H(\cdot, \cdot)$-monotonicity in two Hilbert spaces. A set of the four measurable mappings $x, y, u$, and $v$ is called one solution of Problem 1.

\section{Random Iterative Algorithm}

In order to prove the main results, we need the following lemmas.

Lemma 3.1 (see [23]). Let $H_{1}, f_{1}, g_{1}$, and $A$ be defined as in Problem 1. Let $H_{1}\left(f_{1}, g_{1}\right)$ be $\alpha_{A^{-}}$ strongly monotone with respect to $f_{1}, \beta_{A}$-relaxed monotone with respect to $g_{1}$, where $\alpha_{A}>\beta_{A}$. Suppose that $A: X_{1} \rightarrow 2^{X_{1}}$ is an $H_{1}(\cdot, \cdot)$-monotone set-valued mapping with respect to $f_{1}$ and $g_{1}$, then the resolvent operator $R_{A, \lambda}^{H_{1}(\cdot, \cdot)}=(H(f, g)+\lambda A)^{-1}$ is a single-valued mapping.

Lemma 3.2 (see [23]). Let $H_{1}, f_{1}, g_{1}, A$ be defined as in Problem 1. Let $H_{1}\left(f_{1}, g_{1}\right)$ be $\alpha_{A}$-strongly monotone with respect to $f_{1}, \beta_{A}$-relaxed monotone with respect to $g_{1}$, where $\alpha_{A}>\beta_{A}$. Suppose that $A: X_{1} \rightarrow 2^{X_{1}}$ is an $H_{1}(\cdot, \cdot)$-monotone set-valued mapping with respect to $f_{1}$ and $g_{1}$. Then, the resolvent operator $R_{A, \Lambda}^{H_{1}(\cdot, \cdot)}$ is $1 /\left(\alpha_{A}-\beta_{A}\right)$-Lipschitz continuous.

Remark 3.3. Some interesting examples concerned with the $H_{1}(\cdot, \cdot)$-monotone mapping and the resolvent operator $R_{A, \lambda}^{H_{1}(\cdot, \cdot)}$ can be found in [23].

Lemma 3.4 (see Chang [8]). Let $V: \Omega \times X_{1} \rightarrow C B\left(X_{1}\right)$ be a $D$-continuous random set-valued mapping. Then for any given measurable mapping $u: \Omega \rightarrow X_{1}$, the set-valued mapping $V(\cdot, u(\cdot))$ : $\Omega \rightarrow C B\left(X_{1}\right)$ is measurable. 
Lemma 3.5 (see Chang [8]). Let $V, W: \Omega \rightarrow C B\left(X_{1}\right)$ be two measurable set-valued mappings, and let $\varepsilon>0$ be a constant and $u: \Omega \rightarrow X_{1}$ a measurable selection of $V$. Then there exists $a$ measurable selection $v: \Omega \rightarrow X_{1}$ of $W$, such that for all $t \in \Omega$,

$$
\|u(t)-v(t)\| \leq(1+\varepsilon) D(V(t), W(t)) .
$$

Lemma 3.6. The four measurable mappings $x, u: \Omega \rightarrow X_{1}$ and $y, v: \Omega \rightarrow X_{2}$ are solution of Problem 1 if and only if, for all $t \in \Omega$,

$$
\begin{gathered}
x(t) \in E^{*}(t, u(t)), \\
x(t) \in F^{*}(t, v(t)), \\
p(t, u(t))=R_{A, \lambda}^{H_{1}(\cdot,)}\left[H_{1}\left(f_{1}(p(t, u(t))), g_{1}(p(t, u(t)))\right)-\lambda M\left(s_{M}(t, u(t)), x(t), y(t)\right)\right], \\
q(t, u(t))=R_{B, \rho}^{H_{2}(\cdot, \cdot)}\left[H_{2}\left(f_{2}(q(t, v(t))), g_{2}(q(t, v(t)))\right)-\rho N\left(s_{N}(t, v(t)), x(t), y(t)\right)\right],
\end{gathered}
$$

where $R_{A, \lambda}^{H_{1}(\cdot, \cdot)}=\left(H_{1}\left(f_{1}, g_{1}\right)+\lambda A\right)^{-1}$ and $R_{B, \rho}^{H_{2}(\cdot \cdot)}=\left(H_{2}\left(f_{2}, g_{2}\right)+\rho B\right)^{-1}$ are two resolvent operators.

Proof. From the definitions of $R_{A, \lambda}^{H_{1}(\cdot \cdot)}$ and $R_{B, \rho}^{H_{2}(\cdot \cdot)}$, one has

$$
\begin{aligned}
& H_{1}\left(f_{1}(p(t, u(t))), g_{1}(p(t, u(t)))\right)-\lambda M\left(s_{M}(t, u(t)), x(t), y(t)\right) \\
& \quad \in H_{1}\left(f_{1}(p(t, u(t))), g_{1}(p(t, u(t)))\right)+\lambda A(p(t, u(t))), \quad \forall t \in \Omega, \\
& H_{2}\left(f_{2}(q(t, v(t))), g_{2}(q(t, v(t)))\right)-\rho N\left(s_{N}(t, v(t)), x(t), y(t)\right) \\
& \quad \in H_{2}\left(f_{2}(q(t, v(t))), g_{2}(q(t, v(t)))\right)+\rho B(q(t, v(t))), \quad \forall t \in \Omega .
\end{aligned}
$$

Hence,

$$
\begin{gathered}
0 \in M\left(s_{M}(t, u(t)), x(t), y(t)\right)+A(p(t, u(t))), \quad \forall t \in \Omega, \\
0 \in N\left(s_{N}(t, v(t)), x(t), y(t)\right)+B(q(t, v(t))), \quad \forall t \in \Omega .
\end{gathered}
$$

Thus, $(x, y, u, v)$ is a set of solution of Problem 1 . This completes the proof.

Now we use Lemma 3.6 to construct the following algorithm.

Let $u_{0}: \Omega \rightarrow X_{1}$ and $v_{0}: \Omega \rightarrow X_{2}$ be two measurable mappings, then by Himmelberg [35], there exist $x_{0}: \Omega \rightarrow X_{1}$, a measurable selection of $E^{*}\left(\cdot, u_{0}(\cdot)\right): \Omega \rightarrow C B\left(X_{1}\right)$ and $y_{0}: \Omega \rightarrow X_{2}$, a measurable selection of $F^{*}\left(\cdot, v_{0}(\cdot)\right): \Omega \rightarrow C B\left(X_{2}\right)$. We now propose the following algorithm. 
Algorithm 3.7. For any given measurable mappings $u_{0}: \Omega \rightarrow X_{1}$ and $v_{0}: \Omega \rightarrow X_{2}$, iterative sequences that attempt to solve Problem 1 are defined as follows:

$$
\begin{aligned}
u_{n+1}(t)= & u_{n}(t)-p\left(t, u_{n}(t)\right) \\
& +R_{A, \lambda}^{H_{1}(\cdot, \cdot)}\left[H_{1}\left(f_{1}\left(p\left(t, u_{n}(t)\right)\right), g_{1}\left(p\left(t, u_{n}(t)\right)\right)\right)-\lambda M\left(s_{M}\left(t, u_{n}(t)\right), x_{n}(t), y_{n}(t)\right)\right], \\
v_{n+1}(t)= & v_{n}(t)-q\left(t, v_{n}(t)\right) \\
& +R_{B, \rho}^{H_{2}(\cdot,)}\left[H_{2}\left(f_{2}\left(q\left(t, v_{n}(t)\right)\right), g_{2}\left(q\left(t, v_{n}(t)\right)\right)\right)-\rho N\left(s_{N}\left(t, v_{n}(t)\right), x_{n}(t), y_{n}(t)\right)\right] .
\end{aligned}
$$

Choose $x_{n+1}(t) \in E^{*}\left(t, u_{n+1}(t)\right)$ and $y_{n+1}(t) \in F^{*}\left(t, v_{n+1}(t)\right)$, such that

$$
\begin{aligned}
& \left\|x_{n+1}(t)-x_{n}(t)\right\|_{X_{1}} \leq\left(1+\varepsilon_{n+1}\right) D\left(E^{*}\left(t, u_{n+1}(t)\right), E^{*}\left(t, u_{n}(t)\right)\right) \\
& \left\|y_{n+1}(t)-y_{n}(t)\right\|_{X_{2}} \leq\left(1+\varepsilon_{n+1}\right) D\left(F^{*}\left(t, v_{n+1}(t)\right), F^{*}\left(t, v_{n}(t)\right)\right)
\end{aligned}
$$

for any $t \in \Omega$ and $n=0,1,2,3, \ldots$

Remark 3.8. The existence of $x_{n}$ and $y_{n}$ is guaranteed by Lemmas 3.4 and 3.5.

\section{Existence and Convergence}

Theorem 4.1. Let $X_{1}$ and $X_{2}$ be two separable real Hilbert spaces. Suppose that $s_{M}, p: \Omega \times X_{1} \rightarrow X_{1}$ and $s_{N}, q: \Omega \times X_{2} \rightarrow X_{2}$ are four random mappings. Suppose that $f_{1}, g_{1}: X_{1} \rightarrow X_{1}, H_{1}:$ $X_{1} \times X_{1} \rightarrow X_{1}, f_{2}, g_{2}: X_{2} \rightarrow X_{2}, H_{2}: X_{2} \times X_{2} \rightarrow X_{2}$ are six single-valued mappings. Assume that

(1) $A: X_{1} \rightarrow 2^{X_{1}}$ is an $H_{1}(\cdot, \cdot)$-monotone with respect to operators $f_{1}$ and $g_{1}$,

(2) $B: X_{2} \rightarrow 2^{X_{2}}$ is an $H_{2}(\cdot, \cdot)$-monotone with respect to operators $f_{2}$ and $g_{2}$,

(3) $p(t, u(t)) \cap \operatorname{dom}(A) \neq \emptyset$ and $q(t, v(t)) \cap \operatorname{dom}(B) \neq \emptyset$ for all $t \in \Omega$,

(4) $M: X_{1} \times X_{1} \times X_{2} \rightarrow X_{1}$ is $\zeta_{A}(t)$-monotone with respect to the mapping $s_{M}$ in the first argument, $\xi_{M}(t)$-Lipschitz continuous with respect to mapping $s_{M}$ in the first argument, $\beta_{M}(t)$-Lipschitz continuous with respect to the second argument and $\eta_{M}(t)$-Lipschitz continuous with respect to the third argument,

(5) $N: X_{2} \times X_{1} \times X_{2} \rightarrow X_{2}$ is $\zeta_{B}(t)$-monotone with respect to the mapping $s_{N}$ in the first argument, $\xi_{N}(t)$-Lipschitz continuous with respect to mapping $s_{N}$ in the first argument, $\beta_{N}(t)$-Lipschitz continuous with respect to the second argument and $\eta_{N}(t)$-Lipschitz continuous with respect to the third argument,

(6) Let $E: \Omega \times X_{1} \rightarrow \mathcal{F}\left(X_{1}\right)$ and $F: \Omega \times X_{2} \rightarrow \mathcal{F}\left(X_{2}\right)$ be two random fuzzy mappings satisfying the condition $(* *), \alpha, \beta, E^{*}$ and $F^{*}$ are four mappings induced by $E$ and $F$. $E^{*}$ and $F^{*}$ are $\xi_{E}(t)$-D-Lipschitz and $\xi_{F}(t)$-D-Lipschitz continuous, respectively;

(7) $p$ is $\delta_{p}(t)$-strongly monotone with respect to its second argument, $\sigma_{p}(t)$-Lipschitz continuous with respect to its second argument, $H_{1}\left(f_{1}, g_{1}\right)$ is $\mu_{A}(t)$-strongly monotone 
with respect to the mapping $p$ and $a_{A}(t)$-Lipschitz continuous with respect to the mapping $p$,

(8) $H_{1}\left(f_{1}, g_{1}\right)$ is $\alpha_{A}$-strongly monotone with respect to $f_{1}$, and $\beta_{A}$-relaxed monotone with respect to $g_{1}$, where $\alpha_{A}>\beta_{A}$,

(9) $q$ is $\delta_{q}(t)$-strongly monotone with respect to its second argument and $\sigma_{q}(t)$-Lipschitz continuous with respect to its second argument, $H_{2}\left(f_{2}, g_{2}\right)$ is $\mu_{B}(t)$-strongly monotone with respect to the mapping $q$, and $a_{B}(t)$-Lipschitz continuous with respect to the mapping q,

(10) $\mathrm{H}_{2}\left(f_{2}, g_{2}\right)$ is $\alpha_{B}$-strongly monotone with respect to $f_{2}$ and $\beta_{B}$-relaxed monotone with respect to $g_{2}$, where $\alpha_{B}>\beta_{B}$,

If

$$
\begin{aligned}
A(t)= & \frac{\lambda}{\alpha_{A}-\beta_{A}} \beta_{M}(t) \xi_{E}(t)+\sqrt[2]{1-2 \delta_{p}(t)+\left[\sigma_{p}(t)\right]^{2}} \\
& +\frac{1}{\alpha_{A}-\beta_{A}} \sqrt[2]{1-2 \mu_{A}(t)+\left[a_{A}(t)\right]^{2}} \\
& +\frac{1}{\alpha_{A}-\beta_{A}} \sqrt[2]{1-2 \lambda \zeta_{A}(t)+\lambda^{2}\left[\xi_{M}(t)\right]^{2}}, \\
B(t)= & \frac{\lambda}{\alpha_{A}-\beta_{A}} \eta_{M}(t) \xi_{F}(t), \\
C(t)= & \frac{\rho}{\alpha_{B}-\beta_{B}} \beta_{N}(t) \xi_{E}(t), \\
D(t)= & \frac{\rho}{\alpha_{B}-\beta_{B}} \eta_{N}(t) \xi_{F}(t)+\sqrt[2]{\left.1-2 \delta_{q}(t)+\left[\sigma_{q}(t)\right)\right]^{2}} \\
& +\frac{1}{\alpha_{B}-\beta_{B}} \sqrt[2]{1-2 \mu_{B}(t)+\left[a_{B}(t)\right]^{2}} \\
& +\frac{1}{\alpha_{B}-\beta_{B}} \sqrt[2]{1-2 \rho \zeta_{B}(t)+\rho^{2}\left[\xi_{N}(t)\right]^{2}}, \\
0< & A(t)+C(t)<1, \quad \forall t \in \Omega, \\
0< & B(t)+D(t)<1, \quad \forall t \in \Omega,
\end{aligned}
$$

then there exist four measurable mappings $x, u: \Omega \rightarrow X_{1}$ and $y, v: \Omega \rightarrow X_{2}$, such that $(x, y, u, v)$ is a set of solution of Problem 1. Moreover,

$$
\lim _{n \rightarrow \infty} x_{n}(t)=x(t), \quad \lim _{n \rightarrow \infty} y_{n}(t)=y(t), \quad \lim _{n \rightarrow \infty} u_{n}(t)=u(t), \quad \lim _{n \rightarrow \infty} v_{n}(t)=v(t),
$$

where $x_{n}(t), y_{n}(t), u_{n}(t)$, and $v_{n}(t)$ are defined as in Algorithm 3.7. 
Proof. To simplify calculations, for any $n \in N$, we let

$$
\begin{gathered}
s_{n}(t)=H_{1}\left(f_{1}\left(p\left(t, u_{n}(t)\right)\right), g_{1}\left(p\left(t, u_{n}(t)\right)\right)\right)-\lambda M\left(s_{M}\left(t, u_{n}(t)\right), x_{n}(t), y_{n}(t)\right), \\
t_{n}(t)=H_{2}\left(f_{2}\left(q\left(t, v_{n}(t)\right)\right), g_{2}\left(q\left(t, v_{n}(t)\right)\right)\right)-\rho N\left(s_{N}\left(t, v_{n}(t)\right), x_{n}(t), y_{n}(t)\right), \\
S_{n}(t)=-p\left(t, u_{n}(t)\right)+R_{A, \lambda}^{H_{1}(\cdot,)}\left(s_{n}(t)\right), \quad T_{n}(t)=-q\left(t, v_{n}(t)\right)+R_{B, \rho}^{H_{2}(\cdot, \cdot)}\left(t_{n}(t)\right) .
\end{gathered}
$$

We use $\|\cdot\|_{1}$ to replace $\|\cdot\|_{X_{1}}$ and $\|\cdot\|_{2}$ to replace $\|\cdot\|_{X_{2}}$.

Thus,

$$
\begin{aligned}
& u_{n+1}(t)=u_{n}(t)+S_{n}(t), \\
& v_{n+1}(t)=v_{n}(t)+T_{n}(t) .
\end{aligned}
$$

By the definition of $S_{n}(t), T_{n}(t), s_{n}$, and $t_{n}$, we have the following

$$
\begin{aligned}
\left\|S_{n}(t)\right\|_{1}= & \left\|S_{n-1}(t)+S_{n}(t)-S_{n-1}(t)\right\|_{1} \\
= & \left\|\left[u_{n}(t)-u_{n-1}(t)\right]+S_{n}(t)-S_{n-1}(t)\right\|_{1} \\
\leq & \left\|\left[u_{n}(t)-u_{n-1}(t)\right]-\left[p\left(t, \mathrm{u}_{n}(t)\right)-p\left(t, u_{n-1}(t)\right)\right]\right\|_{1} \\
& +\left\|R_{A, \lambda}^{H_{1}(\cdot,)}\left(s_{n}(t)\right)-R_{A, \lambda}^{H_{1}(\cdot,)}\left(s_{n-1}(t)\right)\right\|_{1^{\prime}} \\
\left\|T_{n}(t)\right\|_{2}= & \left\|T_{n-1}(t)+T_{n}(t)-T_{n-1}(t)\right\|_{2} \\
= & \left\|\left[v_{n}(t)-v_{n-1}(t)\right]+T_{n}(t)-T_{n-1}(t)\right\|_{2} \\
\leq & \left\|\left[v_{n}(t)-v_{n-1}(t)\right]-\left[q\left(t, v_{n}(t)\right)-q\left(t, v_{n-1}(t)\right)\right]\right\|_{2} \\
& +\left\|R_{B, \rho}^{H_{2}(\cdot, \cdot)}\left(t_{n}(t)\right)-R_{B, \rho}^{H_{2}(\cdot, \cdot)}\left(t_{n-1}(t)\right)\right\|_{2} .
\end{aligned}
$$

We first prove that for the two sequences $u_{n}(t)$ and $v_{n}(t)$, there exist two sequences $\left\{A_{n}(t)\right\}$ and $\left\{B_{n}(t)\right\}$ in $[0,1]$, such that

$$
\left\|u_{n+1}(t)-u_{n}(t)\right\|_{1} \leq A_{n}(t)\left\|u_{n}(t)-u_{n-1}(t)\right\|_{1}+B_{n}(t)\left\|v_{n}(t)-v_{n-1}(t)\right\|_{2} .
$$

In fact, for the first term in (4.7), from the $\delta_{p}(t)$-strongly monotonicity and $\sigma_{p}(t)$-Lipschitz continuity of the function $p$, we have the following:

$$
\begin{aligned}
&\left\|\left[u_{n}(t)-u_{n-1}(t)\right]-\left[p\left(t, u_{n}(t)\right)-p\left(t, u_{n-1}(t)\right)\right]\right\|_{1}^{2} \\
&=\left\|u_{n}(t)-\mathrm{u}_{n-1}(t)\right\|_{1}^{2}-2\left\langle p\left(t, u_{n}(t)\right)-p\left(t, u_{n-1}(t)\right), u_{n}(t)-u_{n-1}(t)\right\rangle_{1} \\
&+\left\|p\left(t, u_{n}(t)\right)-p\left(t, u_{n-1}(t)\right)\right\|_{1}^{2} \\
& \leq\left\{1-2 \delta_{p}(t)+\left[\sigma_{p}(t)\right]^{2}\right\}\left\|u_{n}(t)-u_{n-1}(t)\right\|_{1}^{2} .
\end{aligned}
$$


For the second term in (4.7), it follows from Lemma 3.2 that

$$
\begin{aligned}
\left\|R_{A, \lambda}^{H_{1}(\cdot, \cdot)}\left(s_{n}(t)\right)-R_{A, \lambda}^{H_{1}(\cdot, \cdot)}\left(s_{n-1}(t)\right)\right\|_{1} \\
\leq \frac{1}{\alpha_{A}-\beta_{A}}\left\|s_{n}(t)-s_{n-1}(t)\right\|_{1} \\
\leq \frac{1}{\alpha_{A}-\beta_{A}} \| H_{1}\left(f_{1}\left(p\left(t, u_{n}(t)\right)\right), g_{1}\left(p\left(t, u_{n}(t)\right)\right)\right)-\lambda M\left(s_{M}\left(t, u_{n}(t)\right), x_{n}(t), y_{n}(t)\right) \\
\quad-\left[H_{1}\left(f_{1}\left(p\left(t, u_{n-1}(t)\right)\right), g_{1}\left(p\left(t, u_{n-1}(t)\right)\right)\right)-\lambda M\left(s_{M}\left(t, u_{n-1}(t)\right), x_{n-1}(t), y_{n-1}(t)\right)\right] \|_{1} \\
\leq \frac{1}{\alpha_{A}-\beta_{A}} \\
\quad \times\left\{\| u_{n}(t)-u_{n-1}(t)-\left[H_{1}\left(f_{1}\left(p\left(t, u_{n}(t)\right)\right), g_{1}\left(p\left(t, u_{n}(t)\right)\right)\right)\right.\right. \\
\left.\quad-H_{1}\left(f_{1}\left(p\left(t, u_{n-1}(t)\right)\right), g_{1}\left(p\left(t, u_{n-1}(t)\right)\right)\right)\right] \|_{1} \\
\quad+\left\|-u_{n}(t)-u_{n-1}(t)-\lambda\left[M\left(s_{M}\left(t, u_{n}(t)\right), x_{n}(t), y_{n}(t)\right)-M\left(s_{M}\left(t, u_{n-1}(t)\right), x_{n}(t), y_{n}(t)\right)\right]\right\|_{1} \\
\quad+\lambda\left\|M\left(s_{M}\left(t, u_{n-1}(t)\right), x_{n}(t), y_{n}(t)\right)-M\left(s_{M}\left(t, u_{n-1}(t)\right), x_{n-1}(t), y_{n}(t)\right)\right\|_{1} \\
\left.\quad+\lambda\left\|M\left(s_{M}\left(t, u_{n-1}(t)\right), x_{n-1}(t), y_{n}(t)\right)-M\left(s_{M}\left(t, u_{n-1}(t)\right), x_{n-1}(t), y_{n-1}(t)\right)\right\|_{1}\right\} .
\end{aligned}
$$

There are four terms in (4.11). Since $H_{1}\left(f_{1}, g_{1}\right)$ is $\mu_{A}(t)$-strongly monotone and $a_{A}(t)$ Lipschitz continuous with respect to the mapping $p$, then for the first term in (4.11), we can obtain the following

$$
\begin{aligned}
& \left\|u_{n}(t)-u_{n-1}(t)-H_{1}\left(f_{1}\left(p\left(t, u_{n}(t)\right)\right), g_{1}\left(p\left(t, u_{n}(t)\right)\right)\right)+H_{1}\left(f_{1}\left(p\left(t, u_{n-1}(t)\right)\right), g_{1}\left(p\left(t, u_{n-1}(t)\right)\right)\right)\right\|_{1}^{2} \\
& =\left\|u_{n}(t)-u_{n-1}(t)\right\|_{1}^{2}-2\left\langle H_{1}\left(f_{1}\left(p\left(t, u_{n}(t)\right)\right), g_{1}\left(p\left(t, u_{n}(t)\right)\right)\right)\right. \\
& \left.\quad-H_{1}\left(f_{1}\left(p\left(t, u_{n-1}(t)\right)\right), g_{1}\left(p\left(t, u_{n-1}(t)\right)\right)\right), u_{n}(t)-u_{n-1}(t)\right\rangle_{1} \\
& +\left\|H_{1}\left(f_{1}\left(p\left(t, u_{n}(t)\right)\right), g_{1}\left(p\left(t, u_{n}(t)\right)\right)\right)-H_{1}\left(f_{1}\left(p\left(t, u_{n-1}(t)\right)\right), g_{1}\left(p\left(t, u_{n-1}(t)\right)\right)\right)\right\|_{1}^{2} \\
& \leq\left\{1-2 \mu_{A}(t)+\left[a_{A}(t)\right]^{2}\right\}\left\|u_{n}(t)-u_{n-1}(t)\right\|_{1}^{2} .
\end{aligned}
$$

For the second term, Since $M$ is $\zeta_{A}(t)$-monotone with respect to the mapping $s_{M}$ in the first argument and $\xi_{M}(t)$-Lipschitz continuous with respect to mapping $s_{M}$ in the first argument, 
so

$$
\begin{aligned}
& \left\|u_{n}(t)-u_{n-1}(t)-\lambda\left[M\left(s_{M}\left(t, u_{n}(t)\right), x_{n}(t), y_{n}(t)\right)-M\left(s_{M}\left(t, u_{n-1}(t)\right), x_{n}(t), y_{n}(t)\right)\right]\right\|_{1}^{2} \\
& =\left\|u_{n}(t)-u_{n-1}(t)\right\|_{1}^{2}-2 \lambda\left\langle M\left(s_{M}\left(t, u_{n}(t)\right), x_{n}(t), y_{n}(t)\right)\right. \\
& \left.-M\left(s_{M}\left(t, u_{n-1}(t)\right), x_{n}(t), y_{n}(t)\right), u_{n}(t)-u_{n-1}(t)\right\rangle_{1} \\
& +\lambda^{2}\left\|M\left(s_{M}\left(t, u_{n}(t)\right), x_{n}(t), y_{n}(t)\right)-M\left(s_{M}\left(t, u_{n-1}(t)\right), x_{n}(t), y_{n}(t)\right)\right\|_{1}^{2} \\
& \leq\left\|u_{n}(t)-u_{n-1}(t)\right\|_{1}^{2}-2 \lambda \zeta_{A}(t)\left\|u_{n}(t)-u_{n-1}(t)\right\|_{1}^{2}+\lambda^{2}\left[\xi_{M}(t)\right]^{2}\left\|u_{n}(t)-u_{n-1}(t)\right\|_{1}^{2} \\
& \leq\left\{1-2 \lambda \zeta_{A}(t)+\lambda^{2}\left[\xi_{M}(t)\right]^{2}\right\}\left\|u_{n}(t)-u_{n-1}(t)\right\|_{1}^{2} \text {. }
\end{aligned}
$$

For the third term, $M$ is $\beta_{M}(t)$-Lipschitz continuous with respect to the second argument and $E^{*}$ is $\xi_{E}(t)-D$-Lipschitz continuous, therefore, we must have the following:

$$
\begin{aligned}
\| M( & \left.s_{M}\left(t, u_{n-1}(t)\right), x_{n}(t), y_{n}(t)\right)-M\left(s_{M}\left(t, u_{n-1}(t)\right), x_{n-1}(t), y_{n}(t)\right) \|_{1} \\
& \leq \beta_{M}(t)\left\|x_{n}(t)-x_{n-1}(t)\right\|_{1} \\
& \leq \beta_{M}(t)\left(1+\varepsilon_{n}\right) D\left(E^{*}\left(t, u_{n}(t)\right), E^{*}\left(t, u_{n-1}(t)\right)\right) \\
& \left.\leq \beta_{M}(t)\left(1+\varepsilon_{n}\right) \xi_{E}(t) \| u_{n}(t)-u_{n-1}(t)\right) \|_{1} .
\end{aligned}
$$

Similarly, because $\eta_{M}(t)$-Lipschitz continuous with respect to the third argument and $F^{*}$ is $\xi_{F}(t)$ - $D$-Lipschitz continuous, so we can derive

$$
\begin{aligned}
\| M & \left(s_{M}\left(t, u_{n-1}(t)\right), x_{n-1}(t), y_{n}(t)\right)-M\left(s_{M}\left(t, u_{n-1}(t)\right), x_{n-1}(t), y_{n-1}(t)\right) \|_{1} \\
& \leq \eta_{M}(t)\left\|y_{n}(t)-y_{n-1}(t)\right\|_{2} \\
& \leq \eta_{M}(t)\left(1+\varepsilon_{n}\right) D\left(F^{*}\left(t, v_{n}(t)\right), F^{*}\left(t, v_{n-1}(t)\right)\right) \\
& \left.\leq \eta_{M}(t)\left(1+\varepsilon_{n}\right) \xi_{F}(t) \| v_{n}(t)-v_{n-1}(t)\right) \|_{2}
\end{aligned}
$$

If we let

$$
\begin{aligned}
A_{n}(t)= & \frac{\lambda}{\alpha_{A}-\beta_{A}} \beta_{M}(t)\left(1+\varepsilon_{n}\right) \xi_{E}(t)+\sqrt[2]{1-2 \delta_{p}(t)+\left[\sigma_{p}(t)\right]^{2}} \\
& +\frac{1}{\alpha_{A}-\beta_{A}} \sqrt[2]{1-2 \mu_{A}(t)+\left[a_{A}(t)\right]^{2}} \\
& +\frac{1}{\alpha_{A}-\beta_{A}} \sqrt[2]{1-2 \lambda \zeta_{A}(t)+\lambda^{2}\left[\xi_{M}(t)\right]^{2}}
\end{aligned}
$$

and

$$
B_{n}(t)=\frac{\lambda}{\alpha_{A}-\beta_{A}} \eta_{M}(t)\left(1+\varepsilon_{n}\right) \xi_{F}(t)
$$


then, from (4.5) to (4.17), we can have

$$
\left\|u_{n+1}(t)-u_{n}(t)\right\|_{1} \leq A_{n}(t)\left\|u_{n}(t)-u_{n-1}(t)\right\|_{1}+B_{n}(t)\left\|v_{n}(t)-v_{n-1}(t)\right\|_{2}
$$

Under the assumptions of this theorem, in a similar way, we can also show that, for the other two sequences $\left\{v_{n}(t)\right\}$ and $\left\{u_{n}(t)\right\}$, there exist two sequences $\left\{C_{n}(t)\right\}$ and $\left\{D_{n}(t)\right\}$ in $[0,1]$, such that

$$
\left\|v_{n+1}(t)-v_{n}(t)\right\|_{2} \leq C_{n}(t)\left\|u_{n}(t)-u_{n-1}(t)\right\|_{1}+D_{n}(t)\left\|v_{n}(t)-v_{n-1}(t)\right\|_{2}
$$

We now can claim easily that $u_{n}(t), x_{n}(t)$ are two Cauchy sequences in $X_{1}$ and $v_{n}(t)$, $y_{n}(t)$ are two Cauchy sequences in $X_{2}$. In fact, from (4.18) and (4.19), we can obtain the following inequalities:

$$
\begin{aligned}
& \left\|u_{n+1}(t)-u_{n}(t)\right\|_{1}+\left\|v_{n+1}(t)-v_{n}(t)\right\|_{2} \\
& \quad \leq\left(A_{n}(t)+C_{n}(t)\right)\left\|u_{n}(t)-u_{n-1}(t)\right\|_{1}+\left(B_{n}(t)+D_{n}(t)\right)\left\|v_{n}(t)-v_{n-1}(t)\right\|_{2} \\
& \quad \leq \max \left(A_{n}(t)+C_{n}(t), B_{n}(t)+D_{n}(t)\right)\left(\left\|u_{n}(t)-u_{n-1}(t)\right\|_{1}+\left\|v_{n}(t)-v_{n-1}(t)\right\|_{2}\right) .
\end{aligned}
$$

If we let

$$
\begin{gathered}
\theta_{n}(t)=\max \left(A_{n}(t)+C_{n}(t), B_{n}(t)+D_{n}(t)\right), \\
\theta(t)=\max (A(t)+C(t), B(t)+D(t)),
\end{gathered}
$$

then we have the following:

$$
\begin{gathered}
\lim _{n \rightarrow \infty} A_{n}(t)=A(t), \quad \lim _{n \rightarrow \infty} B_{n}(t)=B(t), \quad \lim _{n \rightarrow \infty} C_{n}(t)=C(t), \\
\lim _{n \rightarrow \infty} D_{n}(t)=D(t), \quad \lim _{n \rightarrow \infty} \theta_{n}(t)=\theta(t) .
\end{gathered}
$$

It follows from the assumptions of Theorem 4.1 that $0<\theta(t)<1$, for all $t \in \Omega$, and so $\left\{u_{n}(t)\right\}$ and $\left\{v_{n}(t)\right\}$ are both Cauchy sequences. For sequences $\left\{x_{n}\right\}$ and $\left\{y_{n}\right\}$, since

$$
\begin{aligned}
& \left\|x_{n+1}(t)-x_{n}(t)\right\|_{1} \leq\left(1+\varepsilon_{n+1}\right) D\left(E^{*}\left(t, u_{n+1}(t)\right), E^{*}\left(t, u_{n}(t)\right)\right) \leq 2 \xi_{E}(t)\left\|u_{n+1}(t)-u_{n}(t)\right\|_{1}, \\
& \left\|y_{n+1}(t)-y_{n}(t)\right\|_{2} \leq\left(1+\varepsilon_{n+1}\right) D\left(F^{*}\left(t, v_{n+1}(t)\right), F^{*}\left(t, v_{n}(t)\right)\right) \leq 2 \xi_{F}(t)\left\|v_{n+1}(t)-v_{n}(t)\right\|_{2},
\end{aligned}
$$

thus, $\left\{x_{n}(t)\right\}$ and $\left\{y_{n}(t)\right\}$ are also Cauchy sequences in Hilbert spaces $X_{1}$ and $X_{2}$, respectively. We now show that there exist four measurable mappings $x, u: \Omega \rightarrow X_{1}$ and $y, v$ : $\Omega \rightarrow X_{2}$ such that $(x, y, u, v)$ is a set of solution of Problem 1 and

$$
\lim _{n \rightarrow \infty} x_{n}(t)=x(t), \quad \lim _{n \rightarrow \infty} y_{n}(t)=y(t), \quad \lim _{n \rightarrow \infty} u_{n}(t)=u(t), \quad \lim _{n \rightarrow \infty} v_{n}(t)=v(t),
$$


where $\left\{x_{n}(t)\right\},\left\{y_{n}(t)\right\},\left\{u_{n}(t)\right\}$, and $\left\{v_{n}(t)\right\}$ are four iterative sequences generated by Algorithm 3.7.

Because $X_{1}, X_{2}$ are two Hilbert spaces and $\left\{x_{n}(t)\right\},\left\{y_{n}(t)\right\},\left\{u_{n}(t)\right\}$, and $\left\{v_{n}(t)\right\}$ are four Cauchy sequences, thus, there exist four elements $\{x(t)\},\{y(t)\},\{u(t)\}$, and $\{v(t)\}$ such that

$$
\lim _{n \rightarrow \infty} x_{n}(t)=x(t), \quad \lim _{n \rightarrow \infty} y_{n}(t)=y(t), \quad \lim _{n \rightarrow \infty} u_{n}(t)=u(t), \quad \lim _{n \rightarrow \infty} v_{n}(t)=v(t)
$$

Furthermore,

$$
\begin{aligned}
d\left(x(t), E^{*}(t, u(t))\right) & =\inf \left\{\|x(t)-a\|: a \in E^{*}(t, u(t))\right\} \\
& \leq\left\|x(t)-x_{n}(t)\right\|_{1}+d\left(x_{n}(t), E^{*}(t, u(t))\right) \\
& \leq\left\|x(t)-x_{n}(t)\right\|_{1}+D\left(E^{*}\left(t, u_{n}(t)\right), E^{*}(t, u(t))\right) \\
& \leq\left\|x(t)-x_{n}(t)\right\|_{1}+\xi_{E}(t)\left\|u_{n}(t)-u(t)\right\|_{1} .
\end{aligned}
$$

Since $\lim _{n \rightarrow \infty} x_{n}(t)=x(t), \lim _{n \rightarrow \infty} u_{n}(t)=u(t)$, and $E^{*}(t, u(t)) \in C B\left(X_{1}\right)$, we have the following:

$$
x(t) \in E^{*}(t, u(t))
$$

Similar argument leads to the fact that

$$
y(t) \in F^{*}(t, v(t))
$$

By the continuity of $p, q, H_{1}, f_{1}, g_{1}, H_{2}, f_{2}, g_{2}, E^{*}, F^{*}, R_{A, l}^{H_{1}(\cdot, \cdot)}$, and $R_{B, p}^{H_{2}(\cdot,)}$, we have the following:

$$
\begin{aligned}
& p(t, u(t))=R_{A, \lambda}^{H_{1}(\cdot, \cdot)}\left[H_{1}\left(f_{1}(p(t, u(t))), g_{1}(p(t, u(t)))\right)-\lambda M\left(s_{M}(t, u(t)), x(t), y(t)\right)\right], \\
& q(t, u(t))=R_{B, \rho}^{H_{2}(\cdot, \cdot)}\left[H_{2}\left(f_{2}(q(t, v(t))), g_{2}(q(t, v(t)))\right)-\rho N\left(s_{N}(t, v(t)), x(t), y(t)\right)\right] .
\end{aligned}
$$

So, by Lemma 3.6, $(x, y, u, v)$ is a set of solution to Problem 1 .

This completes the proof.

Remark 4.2. By some suitable choices of mappings in Theorem 4.1, the main results in this paper extend many existing ones, for instance, the main results in $[10,11,21,23,26,31,34]$.

\section{Acknowledgment}

This work was supported by the National Natural Science Foundation of China (10671135). 


\section{References}

[1] C. Baiocchi and A. Capelo, Variational and Quasivariational Inequalities, Application to Free Boundary Problems, John Wiley \& Sons, New York, NY, USA, 1984.

[2] A. Bensoussan, Stochastic Control by Functional Analysis Methods, vol. 11 of Studies in Mathematics and Its Applications, North-Holland, Amsterdam, The Netherlands, 1982.

[3] A. Bensoussan and J.-L. Lions, Impulse Control and Quasivariational Inequalities, Gauthiers-Villers, Bordas, Paris, France, 1984.

[4] S. S. Chang, Variational Inequality and Complementarity Problem Theory with Applications, Shanghai Scientific \& Technology Literature, Shanghai, China, 1991.

[5] S. S. Chang, Variational Inequalities and Related Problems, Chongqing Publishing, Chongqing, China, 2008.

[6] R. U. Verma, "A-monotonicity and applications to nonlinear variational inclusion problems," Journal of Applied Mathematics and Stochastic Analysis, vol. 17, no. 2, pp. 193-195, 2004.

[7] X.-Z. Yuan, "Non-compact random generalized games and random quasi-variational inequalities," Journal of Applied Mathematics and Stochastic Analysis, vol. 7, no. 4, pp. 467-486, 1994.

[8] S. S. Chang, Fixed Point Theory with Applications, Chongqing Publishing, Chongqing, China, 1984.

[9] S. S. Chang and N. J. Huang, "Generalized random multivalued quasi-complementarity problems," Indian Journal of Mathematics, vol. 35, no. 3, pp. 305-320, 1993.

[10] S. S. Chang and N. J. Huang, "Random generalized set-valued quasi-complementarity problems," Acta Mathematicae Applicatae Sinica, vol. 16, pp. 396-405, 1993.

[11] S. S. Zhang and Y. G. Zhu, "Problems concerning a class of random variational inequalities and random quasivariational inequalities," Journal of Mathematical Research and Exposition, vol. 9, no. 3, pp. 385-393, 1989.

[12] N. J. Huang, "Random general set-valued strongly nonlinear quasivariational inequalities," Journal of Sichuan University, vol. 31, no. 4, pp. 420-425, 1994.

[13] N.-J. Huang, "Random generalized set-valued implicit variational inequalities," Journal of Liaoning Normal University, vol. 18, pp. 89-93, 1995.

[14] T. Husain, E. Tarafdar, and X. Z. Yuan, "Some results on random generalized games and random quasi-variational inequalities," Far East Journal of Mathematical Sciences, vol. 2, no. 1, pp. 35-55, 1994.

[15] K.-K. Tan, E. Tarafdar, and X.-Z. Yuan, "Random variational inequalities and applications to random minimization and nonlinear boundary problems," Panamerican Mathematical Journal, vol. 4, no. 2, pp. 55-71, 1994.

[16] N. X. Tan, "Random quasivariational inequality," Mathematische Nachrichten, vol. 125, pp. 319-328, 1986.

[17] N.-J. Huang and Y.-P. Fang, "Generlized $m$-accretive mappings in Banach spaces," Journal of Sichuan University, vol. 38, no. 4, pp. 591-592, 2001.

[18] Y.-P. Fang and N.-J. Huang, "H-monotone operator and resolvent operator technique for variational inclusions," Applied Mathematics and Computation, vol. 145, no. 2-3, pp. 795-803, 2003.

[19] Y. P. Fang, N. J. Huang et al., "Approximate solutions for nonlinear operator inclusions with $(H, \eta)$ monotone operator," Research Report, Sichuan University, Sichuan, China, 2003.

[20] Y.-P. Fang and N.-J. Huang, "H-accretive operators and resolvent operator technique for solving variational inclusions in Banach spaces," Applied Mathematics Letters, vol. 17, no. 6, pp. 647-653, 2004.

[21] Y.-P. Fang, N.-J. Huang, and H. B. Thompson, "A new system of variational inclusions with $(H, \eta)$ monotone operators in Hilbert spaces," Computers \& Mathematics with Applications, vol. 49, no. 2-3, pp. 365-374, 2005.

[22] H.-y Lan, Y. J. Cho, and R. U. Verma, “Nonlinear relaxed cocoercive variational inclusions involving $(A, \eta)$-accretive mappings in Banach spaces," Computers $\mathcal{E}$ Mathematics with Applications, vol. 51, no. 9-10, pp. 1529-1538, 2006.

[23] Y.-Z. Zou and N.-J. Huang, "H(.,.)-accretive operator with an application for solving variational inclusions in Banach spaces," Applied Mathematics and Computation, vol. 204, no. 2, pp. 809-816, 2008.

[24] L. A. Zadeh, "Fuzzy sets," Information and Computation, vol. 8, pp. 338-353, 1965.

[25] N.-J. Huang, "Random completely generalized strongly nonlinear quasi-complementarity problems for random fuzzy mappings," Journal of Fuzzy Mathematics, vol. 5, no. 3, pp. 605-616, 1997.

[26] N.-J. Huang, "Random generalized nonlinear variational inclusions for random fuzzy mappings," Fuzzy Sets and Systems, vol. 105, no. 3, pp. 437-444, 1999. 
[27] R. Ahmad and F. F. Bazán, "An iterative algorithm for random generalized nonlinear mixed variational inclusions for random fuzzy mappings," Applied Mathematics and Computation, vol. 167, no. 2, pp. 1400-1411, 2005.

[28] R. Ahmad and A. P. Farajzadeh, "On random variational inclusions with random fuzzy mappings and random relaxed cocoercive mappings," Fuzzy Sets and Systems, vol. 160, no. 21, pp. 3166-3174, 2009.

[29] Q. H. Ansari and J.-C. Yao, "Systems of generalized variational inequalities and their applications," Applicable Analysis, vol. 76, no. 3-4, pp. 203-217, 2000.

[30] Y. J. Cho, N. J. Huang, and S. M. Kang, "Random generalized set-valued strongly nonlinear implicit quasi-variational inequalities," Journal of Inequalities and Applications, vol. 5, no. 5, pp. 515-531, 2000.

[31] Y. J. Cho and H.-y. Lan, "Generalized nonlinear random $(A, \eta)$-accretive equations with random relaxed cocoercive mappings in Banach spaces," Computers $\mathcal{E}$ Mathematics with Applications, vol. 55, no. 9, pp. 2173-2182, 2008.

[32] N. J. Huang and Y. J. Cho, "Random completely generalized set-valued implicit quasi-variational inequalities," Positivity, vol. 3, no. 3, pp. 201-213, 1999.

[33] N.-J. Huang, X. Long, and Y. J. Cho, "Random completely generalized nonlinear variational inclusions with non-compact valued random mappings," Bulletin of the Korean Mathematical Society, vol. 34, no. 4, pp. 603-615, 1997.

[34] Y.-Z. Zou and N.-J. Huang, "A new system of variational inclusions involving $H(\cdot, \cdot)$-accretive operator in Banach spaces," Applied Mathematics and Computation, vol. 212, no. 1, pp. 135-144, 2009.

[35] C. J. Himmelberg, “Measurable relations," Fundamenta Mathematicae, vol. 87, pp. 53-72, 1975. 